\title{
Enhanced DAB for Efficiency Preservation using Adjustable-Tap High-Frequency Transformer
}

\author{
Armin Jafari, Mohammad Samizadeh Nikoo, Furkan Karakaya and Elison Matioli, Member, IEEE
}

\begin{abstract}
Efficient DC-DC power-conversion with wide-span voltage-regulation is crucial to a sustainable and robust power electronics system. Dual-active-bridge (DAB) offers straightforward regulation and its transformer enables voltage stepup/down required for many applications, such as battery chargers and bus converters for DC distribution systems. However, losing soft-switching at light loads or when operating at voltage gains far from the turns ratio severely degrades the efficiency of DAB, especially at high switching frequencies. In this work, we demonstrate an enhanced DAB (E-DAB) topology which employs an adjustable-tap transformer to extend the softswitching over wider voltage gains and increase the powertransfer capability. By a proper tap adjustment and with single phase-shift modulation, the proposed GaN-based converter achieved a peak efficiency of $97.4 \%$ with an overall efficiency greater than a conventional DAB for voltage gains of up to 2.8 times higher. Employing a quasi-planar matrix transformer with integrated leakage inductance at $300 \mathrm{kHz}$ allowed for an extremely high power density of $10 \mathrm{~kW} / \mathrm{l}(7.5 \mathrm{~kW} / \mathrm{l}$ with cooling). The tapped transformer did not incur extra losses to the topology. The gain versus power-transfer characteristic for softswitching operation was derived for the E-DAB and its improvement in efficiency was experimentally verified over a wide power range.
\end{abstract}

Index Terms-DAB, E-DAB, Variable-tap transformer, Wide voltage-gain, Tap-changer, Soft-switching, High frequency, Efficiency preservation, GaN, High power density, High efficiency.

\section{INTRODUCTION}

A dual active bridge (DAB) converter can be regarded as a regulated DC transformer, capable of adjusting its output voltage under varying load and input voltage conditions. It controls the power flow bi-directionally and provides isolation, which is important for safety and protection schemes. Thus, DAB is of great interest for a wide range of applications, including electric vehicle chargers, avionics, uninterruptable power supplies, DC micro-grid distribution networks, converters for renewable energy harvesting and telecommunications [1]-[4]. The power transfer in DAB is inductive, in which the transformer series inductance is responsible for power transfer and achieving soft-switching, and the turns ratio of the transformer provides the desired voltage step-up/down.

The large size of these magnetic components limits the power density at low switching frequency therefore increasing

The authors are with the Power and Wide-band-gap Electronics Research Laboratory (POWERlab), École polytechnique fédérale de Lausanne (EPFL), CH-1015 Lausanne, Switzerland (e-mail: armin.jafari@epfl.ch elison.matioli@epfl.ch). switching frequency would significantly reduce the overall the size of DAB as well as its core loss [5].

GaN technology offers smaller gate-capacitance and ONresistance. Improvements in dynamic ON-resistance [6] and Coss-related losses [7], [8] promise efficient operation of softswitched DAB at very high frequencies, with the aforementioned advantage of shrinking passive components. However, maintaining soft-switching is critical for a highfrequency $\mathrm{DAB}$, because hard-switching losses scale up with frequency and cause not only drastic drop in efficiency, but also risk of thermal runaway and device failure. Several methods have been proposed in the literature to extend the soft-switching region of DAB. Among them, increasing the dead-time could improve the soft-switching at light loads at the cost of degrading the efficiency at heavy loads; moreover, when the inductor current reaches zero, increasing dead-time does not help at all, leading to more losses [9]. Various modulation schemes could also broaden the region for softswitching [9]-[12], but the laborious calculations involved in those methods are difficult to be performed at high switching frequency due to the limited computation and time-resolution in controllers and drivers. Another solution is to utilize resonance to extend the soft-switching region by adding extra components, passive or active (not lossless), which add to the design size and complexity [10], [12], [13] and lead to electromagnetic interference issues as the increased gain is based on frequency modulation [14]. DC blocking capacitor together with a new modulation scheme is proposed in [15] and the method could successfully increase efficiency for voltage gains around 2 p.u. but deteriorates the performance under normal operating conditions. The improved performance depends on the proper choice of the blocking capacitor and the B-H curve of the transformer is prone to shifting which brings forward the need to deploy an extra saturation prevention algorithm [16]. Combining DAB with elementary buck and boost converters as a composite converter is proposed in [17], achieving outstanding light-load efficiency extension; however, it comes at the price of losing the galvanic isolation and is suited when the difference between input and output voltage levels is relatively small. The increased complexities in control make this approach less applicable at high frequencies. In [18], a planar transformer with two taps is used to successfully extend the soft-switching range and reduce circulating currents in DAB. However, due to the small leakage inductance in planar transformers [5], [19], an external inductor has to be added in series with the

$$
\text { This work was supported in part by the Swiss Office of Energy under Grant SI/501887-01 (MEPCO). }
$$


transformer at high-voltage side, which limits the converter power density, as designing small efficient inductors at high frequency is challenging [20]. Different tap-changer mechanisms are studied in [21]. Simulation shows the advantage of using tap changer for lower semiconductor loss and smaller transformer current and measurements at voltage gains equal to the nominal tap value supports the generality without investigating different gain conditions.

In this work, we demonstrate an enhanced DAB (E-DAB) to increase the power-transfer capability and broaden the voltage gain under which the converter still maintains softswitching without adding lossy components or complicating the modulation. By introducing a tapped quasi-planar matrix transformer with integrated leakage inductance, a high power density converter is realized, as no external inductors are required. A gain versus power-transfer analysis is presented to characterize the behavior of the E-DAB for efficient operation, which is of great significance to design a controller system for such converters. The proposed analysis is experimentally verified by comparing the E-DAB to a conventional DAB under the same operating conditions. The E-DAB is suitable for many power applications with large variations in load and source power, such as photovoltaics, wind power.

\section{Methodology}

Power transfer for single-phase $\mathrm{DAB}$ when single phase shift (SPS) modulation is employed, can be expressed as

$$
P=\frac{V_{\mathrm{IN}} V_{\mathrm{OUT}}\left(\frac{\varphi}{\pi}\right)\left(1-\frac{\varphi}{\pi}\right)}{2 n f_{\mathrm{SW}} L_{r}}
$$

in which $V_{\mathrm{IN}}$ and $V_{\mathrm{OUT}}$ are DC link voltages for primary and secondary respectively, $\varphi$ is the phase shift between them, $f_{s w}$ is switching frequency, $L_{r}$ and $n$ are the leakage inductance and turns ratio of the transformer, respectively. As formulated in (1), changing $n$ not only can control the power flow (and hence regulate the voltage), but is also an important parameter determining the soft-switching region, under which the transistors operate with the least stress and power dissipation. To achieve soft-switching, the current through $L_{r}$ should be large enough to discharge the output capacitance of the switches in the bridge, which means that the actual gain $\mathrm{G}$ of the DAB should stay within the defined constraints

$$
n\left(1-\frac{2 \varphi}{\pi}\right)<G<\frac{n}{1-\frac{2 \varphi}{\pi}}
$$

The limits of (2) applied to DAB with turns ratio $n$ is illustrated in Fig. 1a in which the shaded area shows the softswitching region. Fig. $1 \mathrm{~b}$ shows the corresponding limits for an E-DAB which is capable of varying its turns ratio between $0.5 n$ and $1.5 n$. a broader the soft-switching region is obtained over wider gain ranges, especially at lower phase shifts, which is the equivalent of light-load operation in which the normal DAB suffers from hard-switching. As (1) suggests, the variable turns ratio provides adjustable power-transfer capability for the E-DAB. Utilizing a tapped transformer along with a tap-changer is the only requirement to extend softswitching range and achieve an adjustable power-transfer capability.
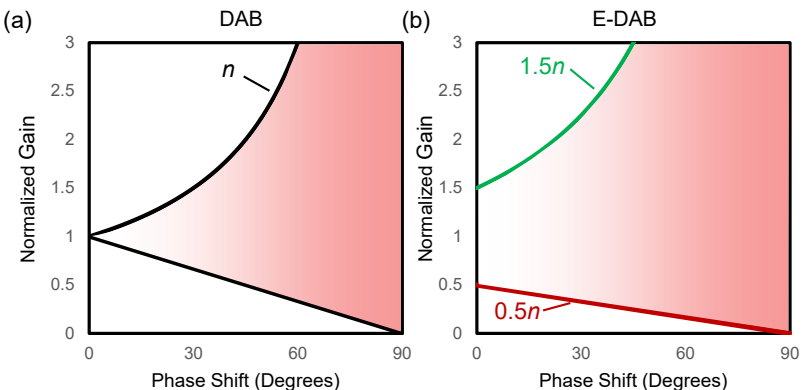

Fig. 1. Normalized gain versus phase shift in (a) DAB and (b) E-DAB. The shaded region represents soft-switching which is significantly extended for the proposed E-DAB especially at lower phase shifts, the equivalent of light-load operation.

\section{TRANSFORMER AND CONVERTER DESIGN}

To examine the proposed method, we designed a matrix transformer equipped with a mechanical tap-changer shown in Fig. 2a. The matrix consists of toroidal cores (R22.1 x13.7x7.9 N49 ferrite material) with Litz wires (1050/\#42 for primary and 66/\#42 for secondary). The ratio $n$ varies between 9 to 12 , with steps of one, providing $+20 /-10 \%$ of adjustment from the nominal value of 1:10. Due to the special winding geometry, transformer's leakage inductance $L_{r}$ is large enough for power transfer and there is no need for adding any external inductors. The change in tap could modify transformer's parameters, especially $L_{r}$, and therefore the predicted soft-switching extension would not be achieved as desired, since (2) assumes a fixed value for $L_{r}$. To verify this, the short-circuit parameters of the transformer were measured with E4990A Impedance Analyzer. As shown in Fig. 2b, increasing the tap value by $33 \%$ from 9 to 12 , modified $L_{r}$ by less than $4 \%$, which guarantees that the theoretical limits still hold. The parasitic $\mathrm{AC}$ resistance of the windings, presented in Fig. 2c, does not vary much in the frequency of interest, which ensures that changing the tap is not adding any extra losses to the topology. The E-DAB converter is shown in Fig. 3a for which $\mathrm{GaN}$ transistors EPC2031 and GS66502B were used for the primary and secondary bridges, respectively, (as demonstrated in Figs. $3 \mathrm{~b}, \mathrm{c})$. We designed a four-layer PCB with minimized gate and power loop inductances for safe performance of the transistors. Ceramic capacitors are placed beside and on the bottom side of each leg in the full-bridges. Transistors are driven by SI8271 isolated gate-drivers with dead-time values of $20 \mathrm{~ns}$ and $40 \mathrm{~ns}$ for primary and secondary switches, respectively. PWM signals from TMS320F28379D DSP are connected to the converter using shielded cables with MMCX connectors. and 5-V isolated DC/DC converters on the bottom layer supply the gate drivers. This combination allowed us to design the transformer to operate at $300 \mathrm{kHz}$ for small footprint as well as reduced core loss due to the inverse relation between switching frequency and magnetic flux density $B$ [5]. The auxiliary power and cooling are provided externally. The tap-changer mechanism in this design is realized using small electromagnetic (EM) relays, capable of switching $2 \mathrm{~A}$. The taps are placed at the secondary side with low current and high voltage. For better lifetime and faster response time, one could use solid-state tap changers, however, the efficiency might be slightly lower [21]. 

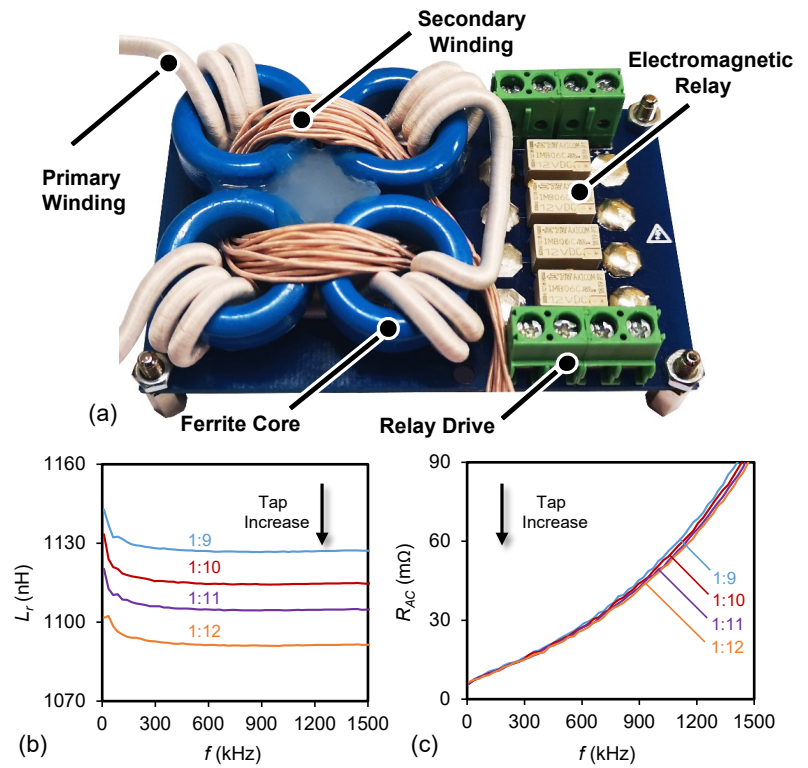

Fig. 2. (a) High-frequency matrix transformer with tap-changer and taps varying between 1:9 to 1:12. (b) leakage inductance $\mathrm{Lr}$ and (c) AC resistance $\left(R_{A C}\right)$ of the transformer measured with E4990A Keysight Impedance Analyzer. The variation of $\operatorname{Lr}$ is less than $4 \%$ over frequency and $R_{A C}$ is not affected by changing the taps in the frequency of interest.

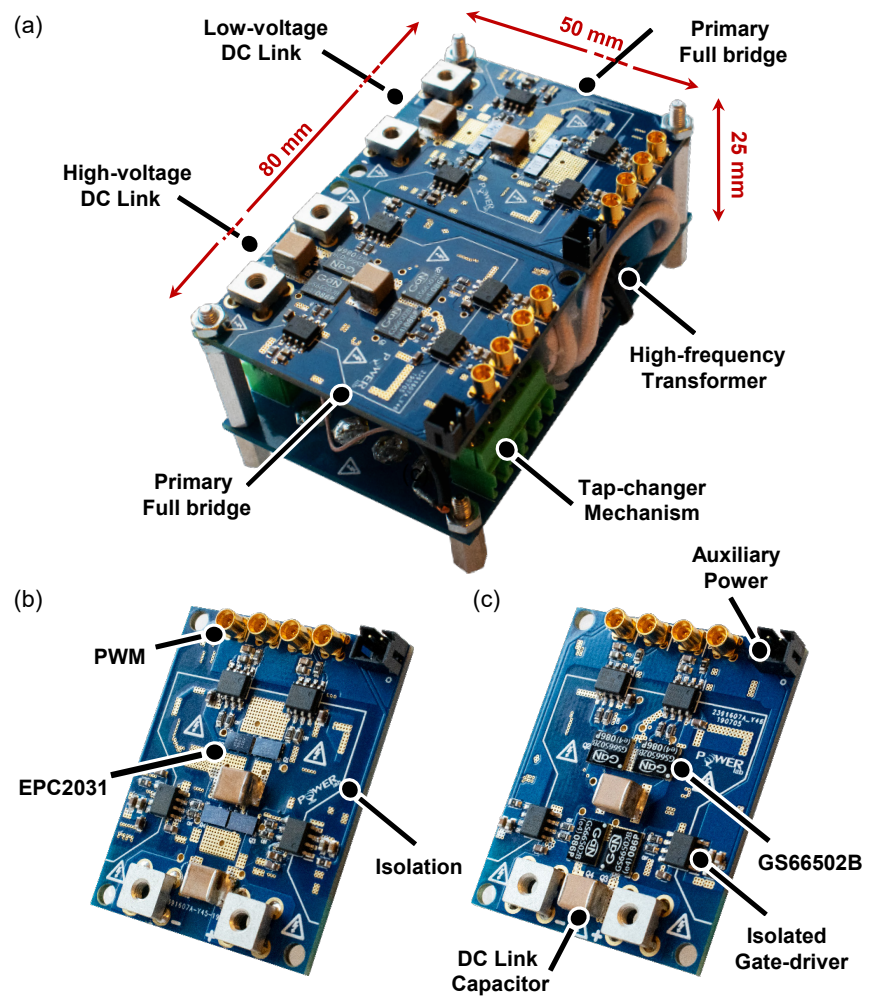

Fig. 3. (a) E-DAB converter with high-frequency variable-tap transformer. (b) Primary full bridge and (c) secondary full bridge utilize GaN technology.

\section{EXPERIMENTAL RESULTS AND ANALYSIS}

Using SPS modulation resulted in the generation of squarewave voltages on the transformer primary and secondary as illustrated in Fig. 4. The output voltage of the converter was regulated at $400 \mathrm{~V}$ by changing $\varphi$ together with $n$ at a constant load. Under these conditions, the efficiency of the DAB for

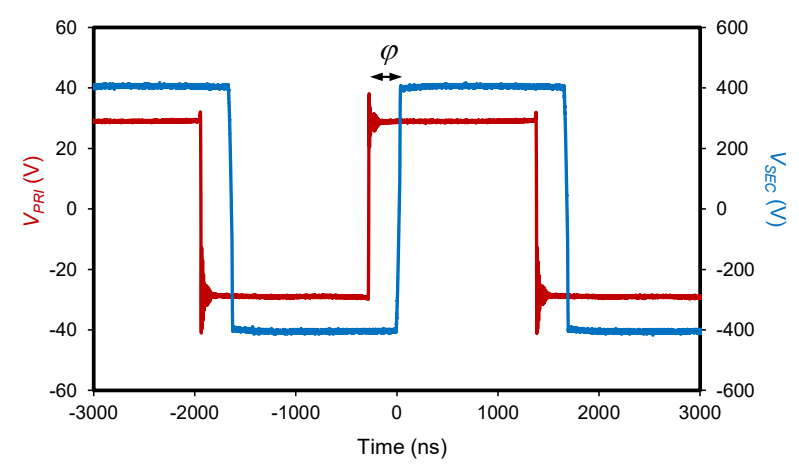

Fig. 4. Primary voltage $\left(V_{P R I}\right)$ and secondary voltage $\left(V_{S E C}\right)$ waveforms of the high-frequency matrix transformer operating with SPS modulation at 300 $\mathrm{kHz}$. $\varphi$ determines the level of transferred power as well as its direction.

different tap values was measured for different input powers (Fig. 5) while the input voltage was matched with the nominal gain of each tap, i.e. output voltage divided by $n$. The power transfer at each tap is a function of $\varphi$, and the input voltage in which the converter operates efficiently could be significantly extended using proper turns ratio. Furthermore, when a high power-transfer is required and even maximum $\varphi$ cannot be sufficient to reach the required target power, changing turns ratio increases the power-transfer capability of the converter, as indicated by (1). EM relays can be actuated under different load conditions as they are able to conduct and block current in both directions [22]. However, in many applications such as battery charging and renewable energy harvesting, the power transfer could be interrupted momentarily to provide lowstress operation of EM relays for improving their lifetime. We compared the efficiency of the DAB with E-DAB at the same input and output voltage conditions, and at a relatively lightload of $200 \mathrm{~W}$ at the output (Fig. 6). The DAB converter was realized by keeping the EM relay at tap 10 closed $(\mathrm{ON})$ all the time, when the other EM relays are open (OFF). This tap was selected as it provides the step up required for nominal operation of the converter between $40-\mathrm{V}$ and $400-\mathrm{V}$ DC-links, under which the transformer core loss is small. Furthermore, we could compare this tap with higher $(11,12)$ and lower (9) taps in the E-DAB, for demonstrating the performance at both ranges. In Fig. 6, E-DAB was operated with larger taps for the lower-end input voltages and for higher input voltages, smaller taps were used. Both converters overlap when E-DAB utilizes turns ratio equal to that of the DAB, i.e. 10. For all the rest of the input voltages, the E-DAB outperformed DAB in terms of efficiency and the peak efficiency was boosted from $96.5 \%$ in DAB to $97.4 \%$ in E-DAB for $30 \%$ higher voltage gain and at a light load of $200 \mathrm{~W}$, equivalent to $20 \%$ of the E-DAB powertransfer capability.

Fig. 6 also shows that E-DAB preserved the higher efficiency down to $14 \mathrm{~V}$ of input voltage, which corresponds to a gain 2.8 times higher than the nominal gain of DAB. The E-DAB is not only better for increasing the efficiency, but also reducing losses in the switches mitigates concerns about thermal runaway with the same cooling effort. The E-DAB converter does not require cooling over a wide range of power and gain, as it operates highly efficient. At $300 \mathrm{kHz}$, power capability of the E-DAB is $1 \mathrm{~kW}$, resulting in a power density of $10 \mathrm{~kW} / 1$ (7.5 kW/1 including forced-air cooling and gate driver supply). Due to the extension of soft-switching, the EMI performance 


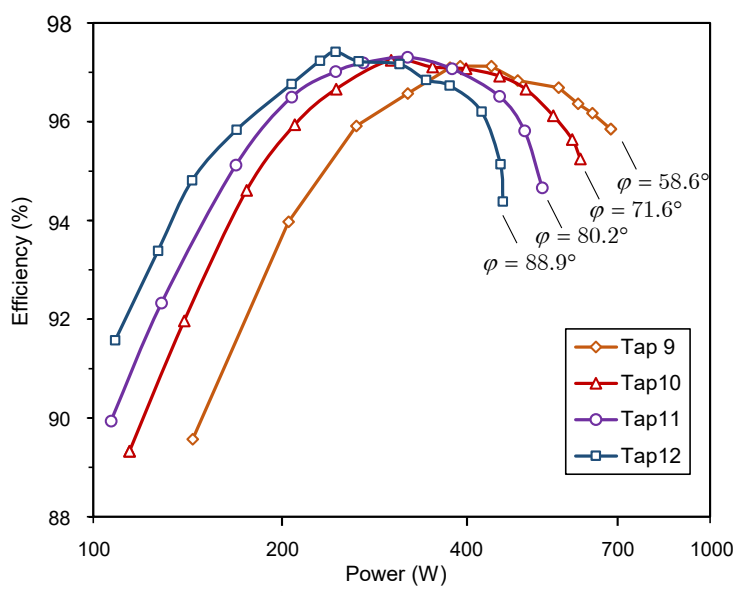

Fig. 5. Efficiency of E-DAB versus its input power for different taps at a fixed output voltage of $400 \mathrm{~V}$ and the gain equal to the tap value. Tap value determines power transfer and increases the power capability when the phase shift reaches its theoretical limit for transferring more power, i.e. $90^{\circ}$. The proper tap selection leads to increase of efficiency at light and heavy loads.

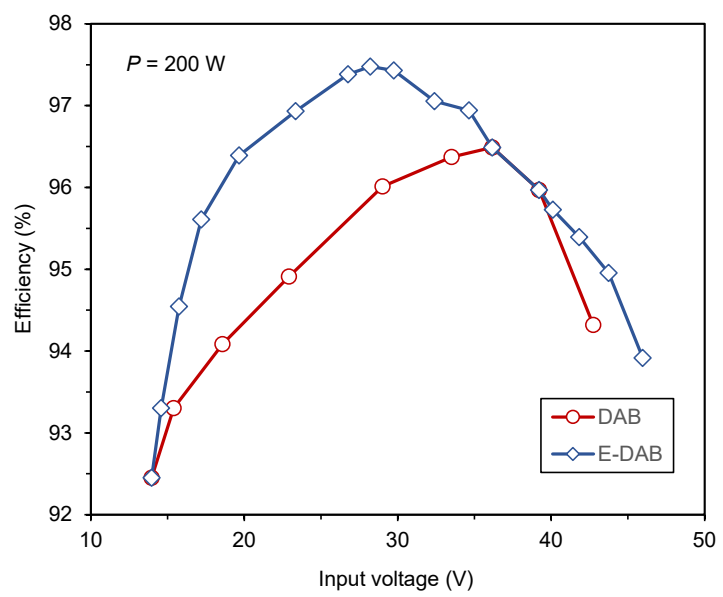

Fig. 6. Efficiency comparison between DAB and E-DAB when a constant load of $200 \mathrm{~W}$ is supplied at $400 \mathrm{~V}$ at the output. The E-DAB outperforms the $\mathrm{DAB}$ over the whole input voltage sweep. This translates into less stress and risk of thermal runaway for the switches by significantly shrinking the total loss up to almost $50 \%$ at the same output power and input/output voltage conditions.

of E-DAB is better, which again is of great importance for high-frequency converter design based on GaN. For example, hard-switching leads to higher voltage ringing on the gatedrive loop and with narrow gate breakdown voltage of GaN devices, there is a risk of device failure at its gate. Fig. 7 shows the drain-to-source and gate-to-source voltages of the primary low-side switches under the same load of $200 \mathrm{~W}$ but different input voltages, during the turn $\mathrm{ON}$ transition. Figs. $7 \mathrm{a}, \mathrm{b}$ correspond to $34 \mathrm{~V}$ input voltage, equivalent to a 12-time voltage gain. While the transition in DAB is partially soft, the E-DAB achieves a full zero-voltage-switching (ZVS). Figs. $7 \mathrm{c}$, d show the input voltage of $23 \mathrm{~V}$, equivalent to a gain of 17.4. E-DAB achieves a partial soft-switching while the DAB could not achieve soft-switching even if the dead-time was extended, since the current in the leakage inductance dropped to almost zero. As a result, an overshoot occurs in drain-tosource voltage of Fig. $7 \mathrm{c}$ due to the reverse conduction of the high-side switch, incurring more losses in the DAB.
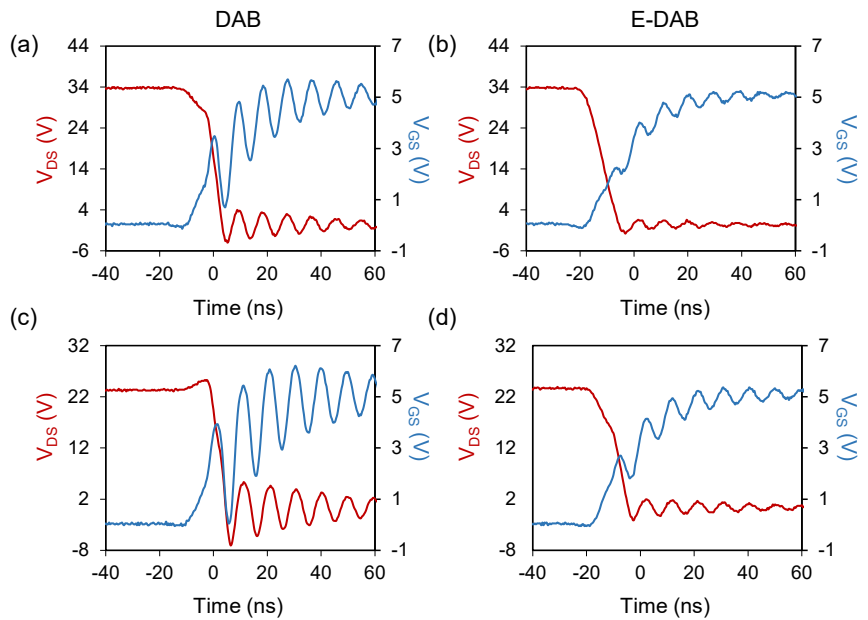

Fig. 7. Turn-ON voltage waveforms of a low-side primary switch. At voltage gain of 12 (a) DAB undergoes partial soft-switching, whereas (b) the same switch in E-DAB is fully soft-switched for the same power. Increasing the gain to 17.4 (c) DAB is hard-switched and the drain-to-source voltage is clamped to DC-Link, incurring extra reverse conduction loss in the high-side switch, while (d) E-DAB still has the capability to be partially soft-switched, which leads to a higher overall efficiency and less switch stress in E-DAB. The waveforms are measured with Tektronix TPP1000 $1 \mathrm{GHz}$ probes.

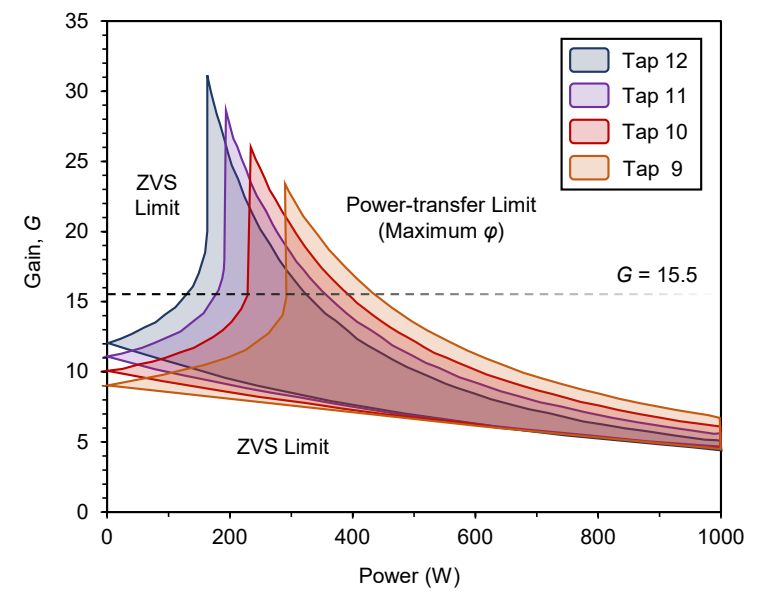

Fig. 8 Voltage gain $(\mathrm{G})$ versus power at which the E-DAB achieves softswitching using different tap values. ZVS limits as well as power-transfer constraint due to maximum phase shift are specified for each tap. E-DAB promises extension of power-transfer capability as well as higher efficiency under light and heavy loads. Experimental results of Fig. 9 verifies the validity of the analysis for $\mathrm{G}=15.5$, in which $\mathrm{E}-\mathrm{DAB}$ outperforms $\mathrm{DAB}$ in terms of efficiency over a wide power range.

Besides the extension of efficiency over wider gain ranges at a fixed power, E-DAB improves the efficiency over wide range of transferred power when a fixed gain is required. For a fixed output voltage, one can solve the limits for $G$ as indicated by (2) for different values of $\varphi$ and use (1) to obtain a gain versus power-transfer characteristic plane for the EDAB. Combined with the power-transfer limit due to maximum $\varphi$, such characteristic specifies the operating range under which E-DAB is soft-switched over different $n$ values. The characteristic plane is presented in Fig. 8 for the E-DAB operating at $400 \mathrm{~V}$ at the output DC-link. We experimentally verified the analysis by comparing the $\mathrm{E}-\mathrm{DAB}$ with $\mathrm{DAB}$ at a fixed voltage gain of 15.5. As shown in Fig. 9 E-DAB preserved higher efficiency compared to the $\mathrm{DAB}$ for the whole power sweep range and boosted the efficiency by $5 \%$ at 


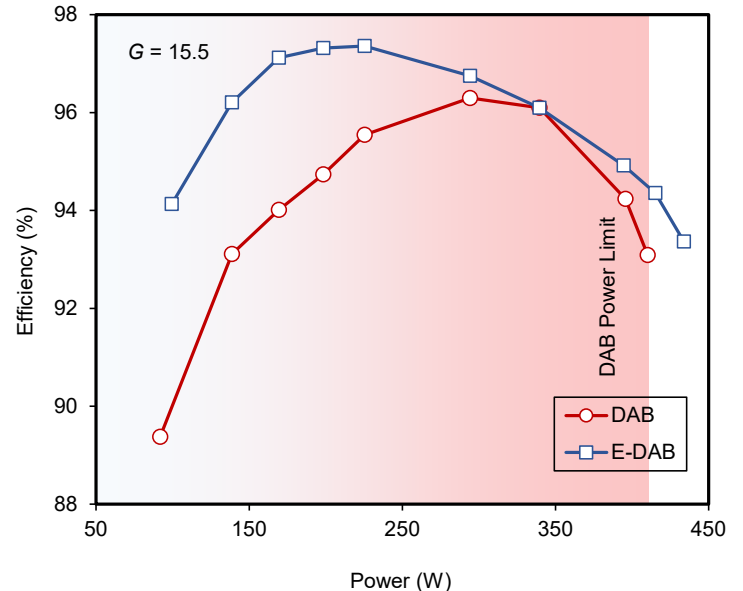

Fig. 9 Efficiency versus power when E-DAB and DAB both operate at a fixed gain of 15.5 at $400 \mathrm{~V}$ output voltage. E-DAB outperforms DAB in terms of efficiency at light and heavy loads, while extending the power-transfer capability. The shaded region indicates the limit for power-transfer capability of the DAB.

an extremely light load of $80 \mathrm{~W}$. Furthermore, when $\varphi$ reached its maximum value in the $\mathrm{DAB}, \mathrm{E}-\mathrm{DAB}$ could still transfer more power. The gain versus power-transfer characteristic is an important analysis tool for design and control of E-DAB converter. The tap values for the transformer design can be selected based on gain and power requirements of target application. Besides, a controller can maximize the efficiency of the E-DAB by selecting proper values for $\varphi$ and $n$ at each operating condition.

\section{CONCLUSION}

In this work we demonstrated an E-DAB topology which employs an adjustable-tap transformer to boost the efficiency over wider voltage gains and provide adjustable powertransfer capability compared to a normal DAB. By designing a quasi-planar matrix transformer whose leakage inductance was used for power transfer and a tap changer at the secondary-side of the transformer, the efficiency of the E$\mathrm{DAB}$ as well as its power density was increased without introducing any extra losses to the converter. The demonstrated converter was realized using GaN transistors and preserved an efficiency always greater than $\mathrm{DAB}$ for up to 2.8 times higher voltage gain at a relatively light load of 200 $\mathrm{W}$ and a high switching frequency of $300 \mathrm{kHz}$. We derived the gain versus power-transfer characteristic for soft-switching operation of the E-DAB which is of great significance for design and control of the E-DAB. The analysis was verified at a fixed gain of 15.5 where the E-DAB outperformed DAB in efficiency and at an extremely light load of $80 \mathrm{~W}, 5 \%$ higher efficiency was achieved. The converter obtained a peak efficiency of $97.4 \%$ and a power-density of $10 \mathrm{~kW} / 1(7.5 \mathrm{~kW} / 1$ including cooling and gate drive supply). E-DAB mitigates the requirement for complex modulation techniques and boosts the global efficiency without adding any extra losses. Due to its better regulation capability and higher efficiency, E-DAB is of great importance for renewable energy harvesting, battery chargers and flexible DC distribution systems.

\section{REFERENCES}

[1] L. Xue, Z. Shen, D. Boroyevich, P. Mattavelli, and D. Diaz, "Dual Active Bridge-Based Battery Charger for Plug-in Hybrid Electric Vehicle With Charging Current Containing Low Frequency Ripple," IEEE Trans. Power Electron., vol. 30, no. 12, pp. 7299-7307, Dec. 2015.

[2] F. Xue, R. Yu, and A. Q. Huang, "A 98.3\% Efficient GaN Isolated Bidirectional DC-DC Converter for DC Microgrid Energy Storage System Applications," IEEE Transactions on Industrial Electronics, vol. 64, no. 11, pp. 9094-9103, Nov. 2017. [3] M. Forouzesh, Y. P. Siwakoti, S. A. Gorji, F. Blaabjerg, and B. Lehman, "StepUp DC-DC Converters: A Comprehensive Review of Voltage-Boosting Techniques, Topologies, and Applications," IEEE Transactions on Power Electronics, vol. 32, no. 12, pp. 9143-9178, Dec. 2017.

[4] T. Liu et al., "Design and Implementation of High Efficiency Control Scheme of Dual Active Bridge Based $10 \mathrm{kV} / 1 \mathrm{MW}$ Solid State Transformer for PV Application," IEEE Trans. Power Electron., vol. 34, no. 5, pp. 4223-4238, May 2019 .

[5] A. Jafari and E. Matioli, "High Step-Up High-Frequency Zero-Voltage Switched GaN-Based Single-Stage Isolated DC-DC Converter for PV Integration and Future DC Grids," at the PCIM Europe 2018, Nuremberg, Germany, 2018.

[6] H. Wang, J. Wei, R. Xie, C. Liu, G. Tang, and K. J. Chen, "Maximizing the Performance of 650-V p-GaN Gate HEMTs: Dynamic RON Characterization and Circuit Design Considerations," IEEE Trans. Power Electron., vol. 32, no. 7, pp. 5539-5549, Jul. 2017.

[7] M. S. Nikoo, A. Jafari, N. Perera, and E. Matioli, "Measurement of LargeSignal $C_{\text {oss }}$ and $\mathrm{C}_{\text {oss }}$ Losses of Transistors Based on Nonlinear Resonance," IEEE Trans. Power Electron., to be published, DOI: 10.1109/TPEL.2019.2938922

[8] M. S. Nikoo, A. Jafari, N. Perera, and E. Matioli, "New Insights on Output Capacitance Losses in Wide-Band-Gap Transistors," IEEE Trans. Power Electron., to be published, DOI: 10.1109/TPEL.2019.2958000.

[9]F. Krismer, "Modeling and Optimization of Bidirectional Dual Active Bridge DC-DC Converter Topologies," ETHZ, 2010.

[10] B. Zhao, Q. Song, W. Liu, and Y. Sun, "Overview of Dual-Active-Bridge Isolated Bidirectional DC-DC Converter for High-Frequency-Link PowerConversion System," IEEE Transactions on Power Electronics, vol. 29, no. 8, pp. 4091-4106, Aug. 2014.

[11] W. Choi, K.-M. Rho, and B.-H. Cho, "Fundamental Duty Modulation of Dual-Active-Bridge Converter for Wide-Range Operation," IEEE Trans. Power Electron., vol. 31, no. 6, pp. 4048-4064, Jun. 2016.

[12] N. Hou and Y. Li, "Overview and Comparison of Modulation and Control Strategies for Non-Resonant Single-Phase Dual-Active-Bridge dc-dc Converter," IEEE Trans. Power Electron., pp. 1-1, 2019.

[13] M. Yaqoob, K. H. Loo, and Y. M. Lai, "Extension of Soft-Switching Region of Dual-Active-Bridge Converter by a Tunable Resonant Tank," IEEE Trans. Power Electron., vol. 32, no. 12, pp. 9093-9104, Dec. 2017.

[14] H.-P. Park, M. Kim, and J.-H. Jung, "Spread-Spectrum Technique Employing Phase-Shift Modulation to Reduce EM Noise for Parallel-Series LLC Resonant Converter," IEEE Trans. Power Electron., vol. 34, no. 2, pp. 1026-1031, Feb. 2019.

[15] Z. Qin, Y. Shen, P. C. Loh, H. Wang, and F. Blaabjerg, "A Dual Active Bridge Converter with an Extended High-Efficiency Range by DC Blocking Capacitor Voltage Control," IEEE Trans. Power Electron., vol. 33, no. 7, pp. 5949-5966, Jul. 2018.

[16] S. A. Assadi, H. Matsumoto, M. Moshirvaziri, M. Nasr, M. S. Zaman, and O. Trescases, "Active Saturation Mitigation in High-Density Dual-Active-Bridge DC-DC Converter for On-Board EV Charger Applications," IEEE Trans. Power Electron., pp. 1-1, 2019.

[17] H. Chen, K. Sabi, H. Kim, T. Harada, R. Erickson, and D. Maksimovic, "A 98.7\% Efficient Composite Converter Architecture with Application-Tailored Efficiency Characteristic," IEEE Trans. Power Electron., vol. 31, no. 1, pp. 101110, Jan. 2016

[18] S. M. Shiva, N. B. Y. Gorla, P. Das, and S. K. Panda, "Tap changing transformer based dual active bridge bi-directional DC-DC converter," in ICPEECCE Asia, Seoul, South Korea, 2015, pp. 2025-2030.

[19] M. A. Saket, N. Shafiei, and M. Ordonez, "LLC Converters with Planar Transformers: Issues and Mitigation," IEEE Trans. Power Electron., vol. 32, no. 6, pp. 4524-4542, Jun. 2017.

[20] R. S. Yang, A. J. Hanson, B. A. Reese, C. R. Sullivan, and D. J. Perreault, "A Low-Loss Inductor Structure and Design Guidelines for High-Frequency Applications," IEEE Trans. Power Electron., vol. 34, no. 10, pp. 9993-10005, Oct. 2019.

[21] S. Taraborrelli, R. Spenke, and R. W. De Doncker, "Bidirectional dual active bridge converter using a tap changer for extended voltage ranges," in EPE'16 ECCE Europe, Karlsruhe, 2016, pp. 1-10.

[22] E. Ramirez-Laboreo, C. Sagues, and S. Llorente, "A New Model of Electromechanical Relays for Predicting the Motion and Electromagnetic Dynamics," IEEE Trans. on Ind. Applicat., vol. 52, no. 3, pp. 2545-2553, May 2016. 